\title{
ORIGINAL ARTICLE \\ Responsiveness and concurrent validity of the revised Capabilities of Upper Extremity-Questionnaire (CUE-Q) in patients with acute tetraplegia
}

\author{
CV Oleson and RJ Marino
}

Study design: This is a longitudinal convenience sample.

Objective: The objective of this study was to evaluate the responsiveness of the revised Capabilities of Upper Extremity-Questionnaire (CUE-Q), in which the item responses were reduced from seven to five levels, relative to the upper extremity motor score (UEMS) and to the self-care subscale of Functional Independence Measure (FIMsc).

Methods: A total of 46 subjects with acute traumatic tetraplegia, 19 motor complete, 27 motor incomplete, completed the revised CUE-Q, UEMS and FIMsc at admission and discharge from rehabilitation.

Results: Subjects were mostly male $(n=42)$ and Caucasian $(n=27)$. The mean age was $44 \pm 21$ years. Predominant etiologies were falls $(n=18)$ and motor vehicle accidents $(n=17)$. During rehabilitation, mean CUE-Q scores increased from $49.8 \pm 30.8$ to $73.7 \pm 36.3$, UEMS increased from $19.6 \pm 11.9$ to $26.3 \pm 13.4$, and FIMsc increased from $9.8 \pm 5.1$ to $21.5 \pm 9.7$. At admission and discharge, CUE scores had excellent to good Spearman correlations $\left(r_{\mathrm{s}}\right)$ with UEMS $\left(r_{\mathrm{s}}=0.89,0.70\right)$ and FIMsc $\left(r_{\mathrm{s}}=0.73,0.80\right)$, but change scores had little to moderate correlations (CUE-UEMS, $r_{\mathrm{s}}=0.07$; CUE-FIMsc, $r_{\mathrm{s}}=0.51$ ), suggesting that the CUE, UEMS and FIM measure related but different constructs. Effect size of the change score was 0.92 for CUE-Q, 0.87 for UEMS and 1.38 for FIMsc. This compares to an effect size of 0.73 for the original 7-level response CUE-Q.

Conclusion: The simplified response set of the CUE-Q maintains the responsiveness of the original version, whereas it increases the ease of use for the patient.

Spinal Cord (2014) 52, 625-628; doi:10.1038/sc.2014.77; published online 3 June 2014

\section{INTRODUCTION}

The Capabilities of Upper Extremity-Questionnaire (CUE-Q) was developed to fill a gap in upper extremity assessment in patients with spinal cord injury (SCI). ${ }^{1}$ The CUE-Q targets the domain of Functional Limitations in the Institute of Medicine model of disablement. ${ }^{2}$ This domain falls between the domains of Impairment and Activity in the International Classification of Functioning Disability and Health ${ }^{3}$ and serves as an important bridge between these two domains. ${ }^{4}$ Functional limitations are actions such as reaching or grasping, rather than tasks or activities such as putting an item on a shelf or using a fork to eat. They reflect the cumulative effect of all impairments on an individual's ability to interact with the environment. For example, a person may have difficulty reaching because of weakness, spasticity, contracture or a combination of all three.

The original version of the CUE-Q asked about limitations in performing certain actions, such as reaching with the right (or left) arm, by using a seven-point scale from one (totally limited, unable to do) to seven (easy, no limitation). Details of the instrument development and the process for selecting items have been described previously. ${ }^{1}$ Briefly, there are 15 items scored separately for the right and left sides, and two items asking about bimanual actions, for a total of 32 items. Arm actions inquire about reaching up, forward and down, pulling and pushing a light and heavy object, extending the wrist and pronating. Hand actions ask about holding items with various grasp patterns: a hammer (cylindrical power grasp), cap of a tube of toothpaste (tripod grasp), key (lateral pinch), the lid of a large jar (wide precision grasp) using the index finger to push a doorbell and manipulating small objects. The bimanual items are lifting a large blanket overhead and pushing down like a push-up weight shift. The instructions ask the respondent to think about the specific part of the arm or hand asked about in each question; if asked about pulling an object, for example, grabbing or holding the object is not required. The CUE-Q is available for download at http://www.spinalcordcenter.org/research/cue.html.

The questionnaire was found to have excellent test-retest reliability, with an interclass correlation coefficient of $0.94 .{ }^{1}$ The CUE-Q is responsive to changes in function after upper extremity tendon transfers and neuroprosthesis implantation. ${ }^{5}$ However, some patients have had difficulty with the seven-level response set, particularly if a sheet listing the responses was not available. In addition, examination of frequency distributions of responses for the CUE-Q items suggested that as many categories were not necessary. As a result, a five-level response set was developed, based on the 
Table 1 Baseline neurological characteristics of subjects

\begin{tabular}{lrr}
\hline Characteristic & N & $\%$ \\
\hline AlS grade & & \\
A & 14 & 31 \\
B & 5 & 11 \\
C & 8 & 17 \\
D & 19 & 41 \\
& & \\
Right motor level & & \\
C1-C4 & 11 & 24 \\
C5 & 25 & 54 \\
C6 & 7 & 15 \\
C7-C8 & 3 & \\
& & \\
Left motor level & & 19 \\
C1-C4 & 9 & 59 \\
C5 & 27 & 11 \\
C6 & 5 & 11 \\
C7-C8 & 5 & \\
\hline
\end{tabular}

Abbreviation: AIS, ASIA Impairment Scale.

International Classification of Functioning Disability and Health qualifiers for capacity and performance. ${ }^{3}$ The revised CUE-Q inquires about difficulty doing an item, with responses ranging from 0 (unable/complete difficulty) to 4 (no difficulty) (See appendix).

The purpose of this study was to determine the responsiveness of the revised CUE-Q and to compare the new five-level version with the original seven-level version. We also evaluated the concurrent validity of the CUE-Q by comparing scores with the Upper Extremity Motor Score (UEMS) and the self-care subscale of the Functional Independence Measure (FIMsc).

\section{MATERIALS AND METHODS}

Using a longitudinal observational repeated measures design, subjects with acute traumatic tetraplegia were recruited from an acute inpatient rehabilitation hospital. All cervical SCI levels and American Spinal Cord Injury (ASIA) Impairment Scale (AIS) grades were eligible, but in order to participate potential subjects with a neurological level of $\mathrm{C} 4$ or above were required to have some upper extremity movement. Patients were excluded, if they had injuries other than SCI affecting their upper extremities or had cognitive deficits that interfered with assessments.

\section{Outcome measures and statistical analysis}

Main outcome measures studied included the UEMS of the International Standards for Neurological Classification of SCI, ${ }^{6}$ the FIMsc, ${ }^{7}$ and the CUE-Q. Data were obtained at admission and discharge from acute inpatient rehabilitation. Physical medicine and rehabilitation physicians examined subjects at admission and discharge to complete the International Standards for Neurological Classification of SCI exam, and rehabilitation staff obtained FIM data by observation, according to the rehabilitation unit procedures. The UEMS and the FIM were selected because they are routinely collected at rehabilitation admission and discharge, and because these measures were used to evaluate concurrent validity of the original CUE-Q. All staff involved in data collection had already completed competency training in this tool as part of their normal work requirements. At the time of data collection, the graded refined assessment of strength, sensibility and prehension was not available. The CUE-Q was conducted via interview by occupational therapists trained in its use.

We calculated the mean and standard deviation, as well as median and interquartile range, for all scores. In addition, right- and left-side scores for
Table 2 Range of scores for outcome measures

\begin{tabular}{|c|c|c|c|c|c|c|}
\hline \multirow[t]{2}{*}{ Measure (max score) } & \multicolumn{3}{|c|}{ Admission } & \multicolumn{3}{|c|}{ Discharge } \\
\hline & Median (IQR) & Low & High & Median (IQR) & Low & High \\
\hline UEMS (50) & $18.5(11-29)$ & 0 & 41 & $29.5(16-36)$ & 0 & 50 \\
\hline CUE-Q (128) & $42.5(30-72)$ & 0 & 115 & $81(48-102)$ & 0 & 125 \\
\hline FIMsc (42) & $6.5(6-13)$ & 6 & 23 & $21(14-30)$ & 6 & 36 \\
\hline
\end{tabular}

Abbreviations: CUE-Q, Capabilities of Upper Extremity-Questionnaire; FIMsc, self-care subscale of the Functional Independence Measure; IQR, interquartile range; UEMS, Upper Extremity Motor Score.

Table 3 Spearman correlation coefficients of CUE-Q with UEMS and FIMsc

\begin{tabular}{|c|c|c|c|c|c|c|}
\hline & \multicolumn{2}{|c|}{ Admission } & \multicolumn{2}{|c|}{ Discharge } & \multicolumn{2}{|c|}{ Change } \\
\hline & UEMS & FIMsc & UEMS & FIMsc & UEMS & FIMsc \\
\hline \multicolumn{7}{|l|}{ CUE-Q } \\
\hline Total & 0.89 & 0.73 & 0.70 & 0.80 & 0.07 & 0.51 \\
\hline Right & 0.80 & & 0.74 & & 0.22 & \\
\hline Left & 0.88 & & 0.71 & & -0.04 & \\
\hline
\end{tabular}

UEMS

$$
0.76
$$

0.73

Abbreviations: CUE-Q, Capabilities of Upper Extremity-Questionnaire; UEMS, Upper Extremity Motor Score; FIMsc, self-care subscale of the Functional Independence Measure.

UEMS and CUE-Q were computed. We determined the correlations among UEMS, FIMsc and CUE-Q scores at admission and discharge from inpatient rehabilitation, as well as the correlations among changes in scores between the two assessment times. Because the scales are ordinal, Spearman correlation coefficients $\left(r_{\mathrm{s}}\right)$ were used. Statistical significance of correlation coefficients is not very informative in this type of analysis because relatively small correlation will have a $P<0.05$. Instead, we used the following interpretation of the size of the correlations: $<0.26$ indicates little to no association, 0.26 to 0.50 indicates fair association, 0.51 to 0.75 indicates moderate to good association, and 0.76 to 1.0 indicates very good to excellent association. ${ }^{8}$ To assess responsiveness of the scales, we compared the effect size of the change score (standardized response mean), defined as the mean change in score divided by the standard deviation of the change score. ${ }^{9}$ All analyses were performed using SAS 9.3 (Cary, NC, USA).

The authors certify that all applicable institutional and governmental regulations concerning the ethical use of human volunteers were followed during the course of this research. The project was approved by the Institutional Review Board at all respective sites of participation.

\section{RESULTS}

We enrolled 65 subjects in the study. Nineteen subjects with missing or incomplete data were excluded from analysis: seven had missing evaluations; three refused discharge testing; three had not been discharged when the project ended; four had incomplete CUE-Q data; and two had incomplete UEMS data. Our final study sample consisted of 46 subjects, 42 of whom were male (91.3\%) with a median age of $44 \pm 21$ years. Twenty-seven subjects $(58.7 \%)$ were Caucasian, 18 (39.2\%) were African-American and the remainder represented other races. The most common etiology of injury were falls $(n=18,39.1 \%)$, followed by motor vehicle collisions $(n=17$, $37.0 \%)$, sports $(n=8,17.4 \%)$ and other traumatic events. Initial AIS grades and motor levels are given in Table 1 . In our sample, the most 
frequent AIS grade was D followed by A, whereas the most common motor level was C5.

Examination of CUE-Q item score distributions found that for every item, all response levels (0-4) were endorsed, with only two scores selected $<5 \%$ of the time. The median and range of scores at admission and discharge are found in Table 2. During inpatient rehabilitation, mean CUE-Q scores improved from $49.8 \pm 30.8$ to $73.7 \pm 36.3$. In this same period mean UEMS increased from $19.6 \pm 11.9$ to $26.3 \pm 13.4$, whereas mean FIMsc scores increased from $9.8 \pm 5.1$ to $21.5 \pm 9.7$.

At admission, the CUE-Q and UEMS demonstrated excellent to very good correlations $\left(r_{\mathrm{s}} \quad 0.80-0.89\right)$ whereas at discharge the correlations were good $\left(r_{\mathrm{s}} 0.70-0.74\right)$ (Table 3 and Figure 1). The CUE-Q and FIMsc scores demonstrated a good correlation at admission and very good correlation at discharge $\left(r_{\mathrm{s}}\right.$ of 0.73 and 0.80). In contrast, the change scores for CUE-Q and UEMS showed little correlation $\left(r_{\mathrm{s}}=0.07\right)$ and change scores for CUE-Q and FIMsc displayed only moderate correlation $\left(r_{\mathrm{s}}=0.51\right)$. The effect size of change was 0.92 for CUE-Q, 0.87 for UEMS and 1.38 for FIMsc.

A few subjects reported levels of difficulty on the CUE-Q inconsistent with UEMS and FIMsc scores. One subject (Figure 1, open circles and arrows) had low admission scores on all measures, but despite minimal change in UEMS and FIMsc reported less difficulty with CUE-Q items at discharge. Another subject (Figure 1, closed circles and arrows) had the opposite pattern: admission CUE-Q scores were high relative to UEMS and FIMsc scores, but at discharge the scores were more congruent.

\section{DISCUSSION}

Our findings indicate that reducing the number of item responses from seven to five did not compromise responsiveness. Notably, the effect size of change scores using the five-level response version of the CUE-Q (0.92) was higher than that using the original seven-level response version (0.73). The correlation of scores at discharge between the revised CUE-Q and UEMS was comparable to that of the original CUE, in which $r_{\mathrm{s}}=0.74$ (unpublished data).

Inconsistencies were found in several patients who reported a level of difficulty with CUE-Q items that were incongruent with their UEMS and FIM scores (Figure 1). Potential reasons for these findings include a subject's misunderstanding of the response set or difficulties in self-evaluation of function in the early weeks post injury. Persons with chronic SCI appear to provide difficulty ratings for CUE-Q items that are more in line with objective measures. In an evaluation of the reliability and concurrent validity of the graded refined assessment of strength, sensibility and prehension, CUE-Q scores demonstrated higher correlation to graded refined assessment of strength, sensibility and prehension subscales than did either SCIM total scores or selfcare subscores. ${ }^{10}$ The recently developed CUE test (CUE-T), ${ }^{11}$ which is an objective counterpart to the CUE-Q, may shed light on the relationship between observed and self-perceived functional limitations.

The moderate correlation of change in FIMsc scores with the UEMS and CUE-Q scores is not surprising, but the low correlation between change in CUE-Q scores and the UEMS was unexpected. Many of the strategies used to improve independence in self-care for persons with complete tetraplegia are adaptive, and thus gains in FIMsc would be expected with little to no improvement in upper extremity strength or functional capabilities. Indeed, the clustering of FIMsc scores at the bottom of the scale on admission to rehabilitation (Figure 1) suggests that the admission FIM is not a good indicator of functional potential. For the CUE-Q and UEMS, the low correlation of change scores could be due to a number of factors. The key muscles in the UEMS represent the myotomes from C5-T1, and an increase in score would not necessarily lead to improved function. An improvement in many muscles from a motor score of 0 to a score of $1-2$ connotes different functional implications than do gains in a few muscles to $\geqslant 3 .{ }^{12}$ In addition, a number of the CUE-Q items (reaching out or up) involve shoulder muscles that are not included
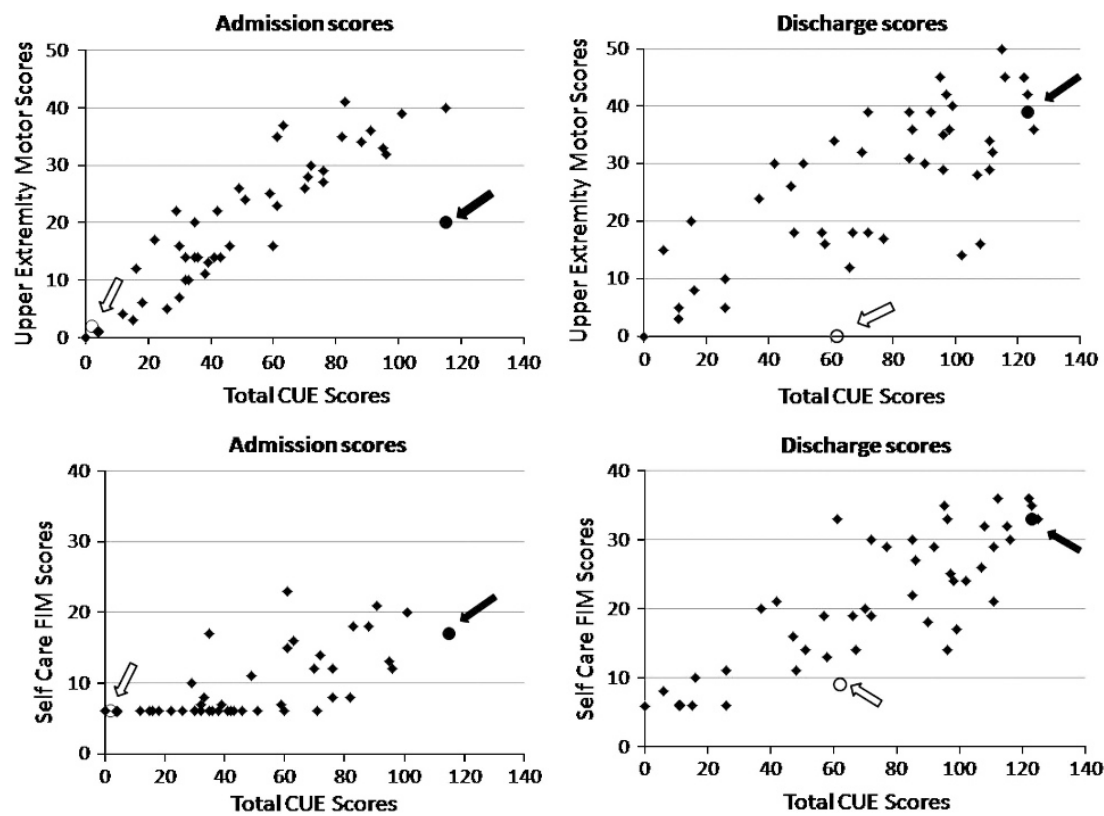

Figure 1 Scatterplots of total CUE-Q scores and upper extremity motor scores (upper graphs) and self-care FIM scores (lower graphs) at admission and discharge from inpatient rehabilitation. Two subjects had unusual patterns of responses. One subject (open circles, open arrows) had CUE-Q scores inconsistent with FIM and UEMS scores at discharge but not at admission; the other subject (closed circles, solid arrows) had inconsistent CUE-Q scores at admission but not at discharge. 
in the UE key muscles, or assess grasp patterns that only roughly correspond to C8 (flexor digitorum profundus of middle finger) and T1 (abductor digiti minimi) key muscles. In some persons, impairments other than strength, such as pain, spasticity or contractures could interfere with the use of the upper limb, which would reduce change in CUE-Q relative to UEMS. Finally, the CUE-Q assesses the perceived difficulty performing specified actions, not actual performance. Newly acquired capabilities may be clumsy or effortful, which could dilute the association with strength measures.

A self-reported functional limitation measure, such as the CUE-Q, offers advantages over measures that focus on hand function in isolation or those that permit the use of adaptive equipment to execute functional activities. Because the CUE-Q separately assesses maneuvers of the entire upper extremity, it can reveal which components of a task are hindering its completion. If the key component actions involved in a task are known, then the responses to CUE-Q items could predict whether an individual would have difficulty performing a task and whether alternative strategies would be helpful. A partial application of mapping actions to tasks was done by Cornwell et al., who identified the movement components for the arm and hand used to complete a representative set of ADL tasks that would be feasible for someone with severe impairments using existing FES technology. ${ }^{13}$ They selected five tasks that included most of the movement components involved in performing 18 tasks to be used as a test battery for evaluating FES and robotic technology. Expanding their list of component movements to include other CUE-Q actions would be worth studying.

There have been a few reported cases where the CUE-Q was useful in identifying functional limitations affecting ADL tasks. In one instance the CUE-Q was felt to be useful in predicting the ability of patients to self-catheterize after continent urinary diversion. ${ }^{14}$ Another study reported a discrepancy between strength assessments and functional assessments during follow-up evaluation for patients who received an upper extremity neuroprosthesis. One patient continued to rate his function on the CUE-Q as 'totally limited' to 'very limited,' despite good functioning of the neuroprosthesis as measured by pinch force. Performance on feeding and grooming tasks showed little improvement with the neuroprosthesis. Further evaluation revealed that this patient was limited by biceps spasticity and by weakness on the opposite extremity, preventing bimanual activity. After addressing these issues via bilateral biceps to triceps transfers, which reduced the impact of biceps spasticity, and via tendon transfers in the non-Freehand arm, which increased function, the use of his neuroprosthesis and satisfaction with its function improved. ${ }^{5}$

\section{CONCLUSION}

The revised CUE-Q retains the responsiveness of the original version, whereas it improves the ease of use for evaluators and patients. The CUE-Q offers a self-reported measure of functional capacity of the arm and hand for persons with tetraplegia. Through its unique design, the revised CUE-Q continues to provide a link between impairment and activity assessments that advances our understanding of the functional limitations facing persons with SCI.

\section{DATA ARCHIVING}

There were no data to deposit.

\section{CONFLICT OF INTEREST}

The authors declare no conflict of interest.

1 Marino RJ, Shea JA, Stineman MG. The Capabilities of Upper Extremity instrument reliability and validity of a measure of functional limitation in tetraplegia. Arch Phys Med Rehabil 1998; 79: 1512-1521.

2 Institute of Medicine. Disability in America: Toward a National Agenda for Prevention. National Academy Press: Washington D.C., USA, 1991.

3 World Health Organization. International Classification of Functioning, Disability and Health: ICF. World Health Organization: Geneva, 2001.

4 Marino RJ. Domains of outcomes in spinal cord injury for clinical trials to improve neurological function. J Rehabil Res Dev 2007; 44: 113-122.

5 Mulcahey MJ, Betz RR, Kozin SH, Smith BT, Hutchinson D, Lutz C. Implantation of the freehand system during initial rehabilitation using minimally invasive techniques. Spinal Cord 2004; 42: 146-155.

6 American Spinal Injury Association. International Standards for Neurological Classification of Spinal Cord Injury, Revised 2000, Reprinted 2002. American Spinal Injury Association: Chicago, IL, 2002.

7 Keith RA, Granger CV, Hamilton BB, Sherwin FS. The functional independence measure: a new tool for rehabilitation. Adv Clin Rehabil 1987; 1: 6-18.

8 Dawson B. Trapp RG. Basic \& Clinical Biostatistics, 4th edn. McGraw-Hill: New York, 2004.

9 Liang MH, Fossel AH, Larson MG. Comparisons of five health status instruments for orthopedic evaluation. Med Care 1990; 28: 632-642.

10 Kalsi-Ryan S, Beaton D, Curt A, Duff S, Popovic MR, Rudhe C et al. The graded redefined assessment of strength sensibility and prehension: reliability and validity. J Neurotrauma 2012; 29: 905-914.

11 Marino RJ, Patrick M, Albright W, Leiby BE, Mulcahey M, Schmidt-Read M et al. Development of an objective test of upper-limb function in tetraplegia: the capabilities of upper extremity test. Am J Phys Med Rehabil 2012; 91: 478-486.

12 Steeves JD, Kramer JK, Fawcett JW, Cragg J, Lammertse DP, Blight AR et al. Extent of spontaneous motor recovery after traumatic cervical sensorimotor complete spinal cord injury. Spinal Cord 2011; 49: 257-265.

13 Cornwell AS, Liao JY, Bryden AM, Kirsch RF. Standard task set for evaluating rehabilitation interventions for individuals with arm paralysis. J Rehabil Res Develop 2012; 49: 395-404

14 Akhavan A, Baker K, Cannon GM, Davies B, Horton JA III, Docimo SG. Pilot evaluation of functional questionnaire for predicting ability of patients with tetraplegia to selfcatheterize after continent diversion. J Spinal Cord Med 2007; 30: 491-496. 